\title{
Medizinische Betreuung inhaftierter Personen am Beispiel der zwangsweisen Rückführungen von Asylsuchenden
}

Christian Kind

Präsident der zentralen Ethikkommission

Schweizerische Akademie der Medizinischen Wissenschaften

Korrespondenz:

Prof. Dr. med. Christian Kind Oberhofstettenstrasse $60 \mathrm{~B}$ CH-9012 St. Gallen

christian.kind[at]bluewin.ch
Seit Erscheinen der medizinethischen Richtlinien zur Ausübung der ärztlichen Tätigkeit bei inhaftierten Personen im Jahr 2002 hat sich die zentrale Ethikkommission (ZEK) der SAMW wiederholt mit Problemen rund um diese Thematik befasst. Jüngstes Beispiel ist die nachfolgend abgedruckte Stellungnahme zu medizinischen Aspekten der sogenannten «Sonderflüge» zur zwangsweisen Rückführung von Asylsuchenden in ihr Herkunftsland. In der Stellungnahme schlägt die ZEK Verbesserungen für die aktuelle Praxis vor. Dass Handlungsbedarf besteht, zeigt auch der auf Initiative des Bundesamtes für Migration angestossene Dialog zwischen den beteiligten ärztlichen und behördlichen Instanzen $\mathrm{zu}$ diesem Themenkreis. Sowohl die FMH als auch die SAMW begrüssen diese Initiative und haben Einsitz in den ensprechenden Arbeitsgruppen.

Die ZEK ist überzeugt, dass die Wahrung des ärztlichen Geheimnisses und die Unabhängigkeit ärztlicher Entscheidungen mit einem geordneten Rechtsvollzug in Einklang gebracht werden können, wenn alle Involvierten gemeinsam nach ethisch vertretbaren und praxistauglichen Lösungen suchen. Sie ist überzeugt, dass die Aufhebung der ärztlichen Schweigepflicht mittels neuer gesetzlicher Ausnahmebestimmungen das für eine medizinische Behandlung notwendige Vertrauensverhältnis zusätzlich erschweren und die Gefahr medizinischer Komplikationen bei Zwangsmassnahmen verschärfen würde. Analoges gilt für die aktuell geführte Diskussion rund um die ärztliche Schweigepflicht betreffend potentiell gefährliche Straftäter. Auch hier ist der Dialog zwischen Gefängnisärzten und Vollzugsbehörden über eine optimale praktische Umsetzung der bestehenden Bestimmungen einer gesetzlichen Neuregelung vorzuziehen.

\section{Zwangsweise Rückführungen: medizinische Aspekte}

2002 hat die Schweizerische Akademie der Medizinischen Wissenschaften (SAMW) Richtlinien zur Ausübung der ärztlichen Tätigkeit bei inhaftierten Personen veröffentlicht. Diese wurden 2012 mit «Hinweisen zur praktischen Umsetzung» ergänzt [1]. Gleichzeitig veröffentlichte die ZEK eine Stellungnahme [2] mit Rahmenbedingungen zur Unterstützung der Einhaltung der Richtlinien, insbesondere

- eine klare Definition der Aufgaben und Verantwortlichkeiten der verschiedenen Akteure (Ärzte [3], Pflegefachpersonen, Gefängnispersonal) bei allen Massnahmen und Entscheidungen, welche die Gesundheit von inhaftierten Personen betreffen;

- die Gewährleistung der fachlichen Unabhängigkeit der in der Medizin im Strafvollzug tätigen Ärzte.
Die ZEK betonte in ihrer Stellungnahme, dass das Prinzip der Gleichwertigkeit der Behandlung (Äquivalenzprinzip) auch während der Ausschaffungshaft Geltung hat. Es muss sichergestellt sein, dass medizinische Untersuchungen und Behandlungen nach den Regeln der ärztlichen Kunst erfolgen können. Dies gilt auch für die Evaluation der Transportfähigkeit von Personen in Ausschaffungshaft. Spezifisch untersagt sind Zwangsbehandlungen, wie z.B. die zwangsweise Verabreichung von Beruhigungsmitteln [4].

Am 6. September 2013 hat die ZEK einen Vertreter der Firma Oseara [5] sowie den Präsidenten der Nationalen Kommission zur Verhütung von Folter [6] zur aktuellen Praxis der zwangsweisen Rückführungen angehört. Sie hat zur Kenntnis genommen, dass laut Oseara die medizinische Begleitung professionalisiert wurde und im Rahmen des aktuel- len Pilotprojekts sichergestellt ist, dass Fachärzte und Rettungssanitäter die zwangsweisen Rückführungen auf dem Luft- und Landweg begleiten.

Mit Sorge hat die ZEK jedoch zur Kenntnis genommen, dass in vielen Fällen Informationen über den Gesundheitszustand der Personen, die zurückgeführt werden, fehlen oder unvollständig sind. Die ZEK hat auch festgestellt, dass nach wie vor in den meisten Kantonen die Gefängnisärzte der Justiz- oder Gefängnisdirektion unterstellt sind. Dies führt dazu, dass Gefängnisärzte in einem Loyalitätskonflikt sind und unter Druck geraten, wenn sie die Transportfähigkeit verneinen oder nicht bereit sind, Informationen zum Gesundheitszustand ohne Einwilligung des Betroffenen weiterzuleiten.

Zur Verbesserung der Situation hat die ZEK vier Punkte formuliert, die bei zwangs- 
weisen Rückführungen $\mathrm{zu}$ beachten sind und nachfolgend erläutert werden:

1. Respektierung der Arzt-Patientenbeziehung bzw. Wahrung des Arztgeheimnisses;

2. Ersatz des Fit-to-Fly-Zertifikats durch Untersuchung auf Kontraindikationen;

3. Verbesserung der Bedingungen für die medizinische Begleitung zwangsweiser Rückführungen;

4. Einhaltung von Rahmenbedingungen für das Auftragsverhältnis mit Organisationen zur Begleitung von zwangsweisen Rückführungen.

\section{Respektierung der Arzt-Patienten- Beziehung bzw. Wahrung des Arztgeheimnisses}

Die Wahrung der Vertraulichkeit ist ein zentrales Element der Arzt-Patienten-Beziehung und muss in jedem Fall nach den gleichen rechtlichen Vorschriften erfolgen, wie sie für Personen in Freiheit gelten. Die Weiterleitung von Gesundheitsinformationen an Kantonale Stellen und das BFM ohne Einverständnis des Patienten und/oder die Untersuchung der betroffenen Person im Hinblick auf mögliche Kontraindikationen für einen bevorstehenden Rückflug unter Zwangsbedingungen ohne deren Wissen und Einverständnis sind nicht mit diesem Grundsatz vereinbar. Beides ist offenbar gängige Praxis.

Aus diesen Gründen muss aus Sicht der ZEK sichergestellt sein, dass das Arztgeheimnis nicht tangiert wird. Wie von der Nationalen Kommission zur Verhütung der Folter und vom Europarat gefordert, müssen unnötige Notfallsituationen vermieden werden. Entsprechend Art. 27 Bundesgesetz über die Anwendung polizeilichen Zwangs und polizeilicher Massnahmen [7] und wie von der Nationalen Kommission zur Verhütung von Folter sowie dem Europäischen Gerichtshof gefordert, sollen die von einer zwangsweisen Rückführung betroffene Person sowie der Arzt, der den Flug begleitet, früh genug, mindestens 72 Stunden vorher, informiert werden, damit allenfalls eine vom Patienten spontan gewünschte oder vom Gefängnisarzt empfohlene medizinische Untersuchung durchgeführt werden kann. Gefängnisärzte sollten ihre Patienten dazu motivieren können, den Ärzten, welche die Rückführung begleiten, ihre medizinischen Daten zur Verfügung zu stellen, insbesondere, wenn eine behandlungsbedürftige
Krankheit vorliegt. Mit Einwilligung des Patienten kann dann der Gefängnisarzt diese Informationen an den Arzt weiterleiten, der den Flug begleitet.

\section{Ersatz des Fit-to-Fly-Zertifikats durch die Untersuchung auf Kontraindikationen}

Gemäss aktueller Praxis stellt der entsendende Kanton ein sogenanntes Fit-to-FlyZertifikat aus. In der Regel wird diese Aufgabe den Gefängnisärzten übertragen; in Einzelfällen werden Fit-to-Fly-Zertifikate offenbar auch von Polizeibeamten ausgestellt. Die aktuelle Praxis ist aus folgenden Gründen nicht akzeptabel:

- Gefängnisärzte unterstehen der ärztlichen Schweigepflicht, Informationen über den Gesundheitszustand des Patienten dürfen nur an Dritte weitergegeben werden, sofern der Patient damit einverstanden ist (vgl. Ziff. 1).

- Es gibt keine evidenzbasierten Kriterien zur Bescheinigung der Transportfähigkeit, sondern es kann lediglich geprüft werden, ob Kontraindikationen vorliegen, die eine zwangsweise Rückführung ausschliessen.

Fit-to-Fly-Zertifikate müssen teilweise zu einem Zeitpunkt (z.B. bei Eintritt in die Institution) ausgestellt werden, der nicht zeitnah zur Rückführung ist. Der Gesundheitszustand einer Person, die rückgeführt werden soll, kann sich verändern.

- Zwangsweise Rückführungen und Fesselungen sind per se mit medizinischen Risiken (Stress, kardiovaskuläre und Atemstörungen, venöse Thrombose) verbunden, die zum Zeitpunkt des Fit-to-FlyZertifikats nicht abgeschätzt werden können.

Aus diesen Gründen fordert die ZEK, auf Fitto-Fly-Zertifikate zu verzichten und diese durch folgende Elemente zu ersetzen:

Personen in Ausschaffungshaft sind grundsätzlich als Risikopatienten zu betrachten. Kontraindikationen sollen in einem individuellen Risikoprofil erfasst werden, dieses muss auch die potentiellen Auswirkungen der Zwangssituation berücksichtigen (Belastungsprofil). Sofern der Patient einverstanden ist, informiert der Gefängnisarzt (oder der behandelnde Arzt) den Arzt, der die zwangsweise Rückführung begleitet, über allfällige Kontraindikationen, die ihm bekannt sind.
- Der Arzt, der die zwangsweise Rückführung begleitet, klärt ab, ob Kontraindikationen vorliegen, und hält diese schriftlich fest. Sofern die betroffene Person einverstanden ist, übergibt er die Bescheinigung der zuständigen Behörde sowie dem Piloten, der den Rückführungsflug durchführt. Für das zeitgerechte Vorliegen und den verbindlichen Inhalt des Belastungsprofils der Rückführung ist die rechtsanwendende Instanz verantwortlich.

\section{Verbesserung der Bedingungen für die medizinische Begleitung zwangsweiser Rückführungen}

Gemäss aktueller Praxis werden Ärzte, die Rückführungen begleiten, vom Bundesamt für Migration (BFM) über den Gesundheitszustand der betroffenen Personen informiert. Die Informationen, die zur Verfügung stehen, sind jedoch oft unvollständig oder fehlen [8]. Informationen zur medizinischen Nachbetreuung sind in der Regel nicht vorhanden. Zudem sind die Kantone nicht immer bereit, rückgeführten Personen Medikamente (z.B. Antihypertensiva, Antidepressiva, Antibiotika, antivirale Medikamente) für eine Überbrückungszeit von einigen Tagen zur Verfügung zu stellen.

Es ist grundsätzlich davon auszugehen, dass zwangsweise Rückführungen mit einem erhöhten gesundheitlichen Risiko verbunden sind, weshalb für alle Personen mindestens eine direkte Begegnung und Befragung durch den begleitenden Arzt erfolgen muss [9]. Falls notwendig, muss ein Übersetzer hinzugezogen werden. Es darf nicht durch Zeitdruck eine künstliche Notfallsituation geschaffen werden. Die verantwortlichen Ärzte müssen genügend Zeit für die seriöse Anamnese vor dem Flug haben und nötige Abklärungen veranlassen können.

Damit die medizinische Beurteilung und Begleitung lege artis durchgeführt werden kann, fordert die ZEK, dass folgende Punkte erfüllt sind:

- Es müssen in jedem Fall die folgenden administrativen Angaben zur Verfügung gestellt werden: Geschlecht, Alter, Zeitpunkt der letzten Mahlzeit. Befindet oder befand (letzte 6 Monate) sich die betroffene Person im Hungerstreik, gehört dies zu den Informationen, die den Begleitärzten zwingend zur Verfügung gestellt werden müssen [10].

- Mit Einverständnis des betroffenen Patienten sollten zudem mindestens die folgenden medizinischen Vorinforma- 
tionen zur Verfügung gestellt werden: aktuelle Beschwerden (inkl. psychische Leiden), Allergien, Medikamente, Past Events, Angaben zum Alkohol- und Zigarettenkonsum, Angaben zur Weiterbehandlung.

- Für die ergänzende Anamnese und klinische Untersuchung für die Rückführung muss genügend Zeit zur Verfügung stehen (mindestens 72 Std., bei Notwendigkeit von Zusatzuntersuchungen entsprechend länger).

- Während der ärztlichen Untersuchung darf die Person nicht gefesselt sein, auch Spucknetze müssen entfernt werden. Die räumlichen Verhältnisse müssen so gestaltet werden, dass das Arztgeheimnis gewahrt werden kann.

- Medizinische Zusatzabklärungen und -untersuchungen können vom untersuchenden Arzt verlangt werden, sofern dies nötig ist.

- Eine medikamentöse Thromboseprävention wird empfohlen, wenn die betroffene Person dies nicht ablehnt.

- Zwangsbehandlungen sind nicht zulässig.

Die ZEK fordert zudem, dass die medizinische Nachbetreuung der rückgeführten Personen so weit wie möglich sichergestellt ist. Dies bedeutet,

- dass Angaben vorliegen zur Weiterbehandlung von rückgeführten Personen, die am Zielort eine unmittelbare engmaschige medizinische Betreuung benötigen;

- dass notwendige Medikamente (z. B. Antibiotika, antivirale Medikamente, Antihypertensiva) für eine Überbrückungszeit von mindestens einer Woche mitgegeben werden.

Die medizinische Verantwortung für die rückgeführten Personen liegt beim Arzt, der die Rückführung begleitet. Er stellt mit Einverständnis des Patienten Rückfragen beim vorbetreuenden (Gefängnis-)Arzt. Problematisch sind Situationen, in welchen die rückgeführten Personen das Gespräch verweigern und der Arzt sich auf den klinischen Eindruck beschränken muss. Es muss gewährleistet sein, dass Ärzte sich auf die
SAMW-Richtlinien berufen und eine medizinische Begleitung verweigern können, wenn eine Kontraindikation für den Transport vorliegt oder die Voraussetzungen für eine adäquate Beurteilung nicht erfüllt sind.

\section{Einhaltung von Rahmenbedingun- gen für das Auftragsverhältnis mit Organisationen zur Begleitung von zwangsweisen Rückführungen}

Im Rahmen eines Pilotversuchs hat das Bundesamt für Migration die medizinische Begleitung von zwangsweisen Rückführungen der Firma Oseara übertragen. Nach Ablauf der Pilotphase, Ende 2013, sollen die Ergebnisse evaluiert werden. Die Beauftragung von privaten Unternehmen wurde vielfach kritisiert.

Aus Sicht der ZEK ist es vertretbar, privaten Unternehmen die medizinische Begleitung von zwangsweisen Rückführungen zu übertragen, wenn die nachfolgenden Bedingungen erfüllt sind:

- Die Firma arbeitet ausschliesslich mit Ärzten zusammen, die qualifiziert sind, diese Aufgabe zu übernehmen. Sie müssen insbesondere auch allfällig auftretende medizinische Notfallsituationen adäquat behandeln können.

- Ärzte, die zwangsweise Rückführungen medizinisch begleiten, müssen Mitglied der FMH sein. Die SAMW-Richtlinien sind Teil der Standesordnung FMH, und bei Nichtbefolgung der Richtlinien können standesrechtliche Massnahmen eingeleitet werden.

- Es muss sichergestellt sein, dass die Dienstleistungen (Abklärung und Begleitung) nach Ansätzen entschädigt werden, die Fehlanreize ausschliessen.

- Es muss gewährleistet sein, dass Ärzte, die Rückführungen begleiten, rechtlich abgesichert sind.

Zentrale Ethikkommission der Schweizerischen Akademie der Medizinischen Wissenschaften (SAMW)

Bern, 18. Oktober 2013. Genehmigt vom Vorstand der Schweizerischen Akademie der Medizinischen Wissenschaften (SAMW), vom Zentralvorstand der Verbindung der Schweizer Ärztinnen und Ärzte (FMH) sowie vom Vorstand der Konferenz Schweizerischer Gefängnisärzte (KSG).

\section{Referenzen}

1 www.samw.ch/de/Ethik/Richtlinien/ Aktuell-gueltige-Richtlinien.html

2 Staatliche Gewalt und Medizinisches Ethos. Stellungnahme der Zentralen Ethikkommission, 2012, www.samw.ch/de/Publikationen/ Stellungnahmen/Aktuell.html

3 Die entsprechenden Texte betreffen immer beide Geschlechter der genannten Personengruppen.

4 Entscheid der Ersten öffentlich-rechtlichen Abteilung des Bundesgerichts vom 20. August 2013, 1B_771/2012, Erwägung 2.3

5 Die Oseara AG begleitet im Auftrag des Bundesamtes für Migration im Rahmen eines Pilotversuchs seit April 2012 zwangsweise Rückführungen auf dem Luft- und Landweg.

6 www.nkvf.admin.ch/

7 Art. 27 Abs. 2 Bundesgesetz über die Anwendung polizeilichen Zwangs und polizeilicher Massnahmen im Zuständigkeitsbereich des Bundes hält Folgendes fest: «Die betroffenen Personen sind vorgängig zu orientieren und anzuhören, soweit der Vollzug selbst dadurch nicht in Frage gestellt wird; es ist ihnen insbesondere Gelegenheit zu geben, dringliche persönliche Angelegenheiten vor der Rückführung zu erledigen oder erledigen zu lassen.»

8 Aus juristischer Sicht heikel ist, dass von den Kantonen eingesetzte Dolmetscher teilweise medizinische Informationen übersetzen, ohne dass eine Entbindung vom Patientengeheimnis vorliegt. Dies trifft auch für Sachbearbeiter des BFM zu, die medizinische Informationen einsehen und weiterleiten.

9 Gemäss Art. 27 ZAG Abs. 3 sind betroffene Personen vor Beginn des Transports ärztlich zu untersuchen, wenn: a) die betroffene Person dies verlangt oder b) Anzeichen für gesundheitliche Probleme feststellbar sind. Vgl. auch «Vorschriften betreffend zwangsweise Rückführungen auf dem Luftweg» Empfehlungen der Konferenz der Kantonalen Justiz- und Polizeidirektorinnen und Polizeidirektoren. Art. 16 (Medizinische Untersuchung) dieser Empfehlungen ist entsprechend anzupassen.

10 Die Information über einen Hungerstreik tangiert das Arztgeheimnis nicht, es handelt sich um eine administrative Information. Diese muss in jedem Fall weitergeleitet werden, das heisst auch dann, wenn die betroffene Person den Gefängnisarzt nicht von der Schweigepflicht entbunden hat. 